\title{
Synthesis of a Novel Family of Polysilsesquioxanes Having Oligothiophenes with Well-Defined Structures
}

\author{
Ichiro Imae, Shotaro Takayama, Daisuke Tokita, Yousuke Ooyama, Kenji Komaguchi, \\ Joji Ohshita, and Yutaka Harima
} Department of Applied Chemistry, Graduate School of Engineering, Hiroshima University, 1-4-1 Kagamiyama, Hiroshima,
Higashi-Hiroshima 739-8527, Japan

Correspondence should be addressed to Ichiro Imae, imae@hiroshima-u.ac.jp

Received 12 June 2012; Accepted 21 October 2012

Academic Editor: Kimihiro Matsukawa

Copyright ( $) 2012$ Ichiro Imae et al. This is an open access article distributed under the Creative Commons Attribution License, which permits unrestricted use, distribution, and reproduction in any medium, provided the original work is properly cited.

Our recent results on the synthesis and properties of a novel family of polysilsesquioxanes having oligothiophenes were reviewed. The polymers anchored on $\mathrm{SiO}_{2}$ or ITO substrates showed excellent mechanical hardness due to the formation of a threedimensional siloxane network structure and chemical linkage between polymer and the surface of metal-oxide substrates. Optical, electrochemical, and electrical properties of polymers were also investigated.

\section{Introduction}

Organic-inorganic hybrid materials have been opening a new field of materials science because of a wide range of possible applications. Among a variety of organic-inorganic hybrid materials, hybrid materials based on a siloxane network are very attractive due to the robust nature of the siloxane bond $[1,2]$ and thus are studied intensively. From the viewpoints of synthetic approach and the resulting materials structure, the siloxane-based hybrid materials can be divided into two classes [3]. Class I corresponds to nanocomposite materials, which are synthesized by an inorganic hydrolytic polycondensation (sol-gel reaction) of tetraalkoxysilane monomer in the presence of low molecular weight organic compounds as dopants. In this class of hybrid materials, the organic dopants are just embedded in the inorganic matrix and are likely to be isolated due to phase separation. Class II corresponds to nanostructured hybrid materials prepared from a solgel reaction of trialkoxysilane-based precursor monomers having organic substituents in the molecular chains. The resulting network polymers are called polysilsesquioxanes (PSQs), where the organic substituents bound covalently to the siloxane network are fixed strongly and homogeneously in the polymer bulk. When the sol-gel reaction occurs on the surface of glass or ITO, terminal silanol groups of PSQs react with hydroxy groups of the metal-oxide surface (Scheme 1), so that the organic functional groups can be fixed on the metal-oxide substrates through the siloxane network in PSQs. PSQs have been used as novel high-performance materials such as water-repellent agents for automobiles, catalyst supports, adsorbents, optics, and biosensors [4-8].

Recently, we introduced oligothiophenes in PSQ for applying PSQs to transparent and conductive films with tunable electrical properties. Oligothiophenes are well studied as photo- and electroactive materials and their electrical conductivities can be changed by chemical or electrochemical oxidation (p-doping) [9-11]. In addition, conductive and energetic properties of oligothiophenes can be controlled by changing a $\pi$-conjugation length or by introducing electron donating or withdrawing groups on the $\pi$-conjugated chains.

In this paper, synthesis of a novel family of polysilsesquioxanes having oligothiophenes (Figure 1) and their electrochemical, optical, electrical, and mechanical properties are described [12-15].

\section{Synthesis of Triethoxysilane Monomers}

2.1. Direct Silylation. We firstly succeeded in the introduction of silyl groups to sexithiophene [13] and octithiophene 

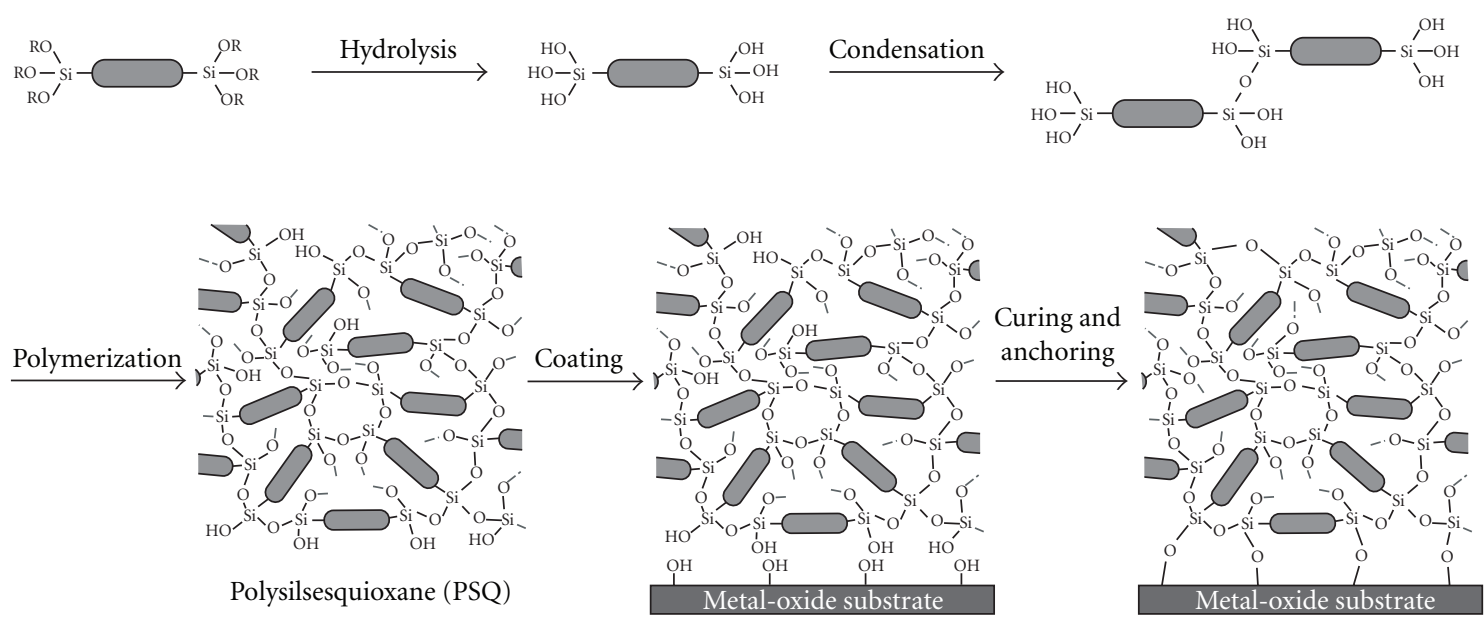

(e.g., glass $\left(\mathrm{SiO}_{2}\right)$, indium tin oxide, titania)

Organic substituents

Scheme 1: Sol-gel reactions of trialkoxysilane monomer and coating of PSQs on metal-oxide substrates.

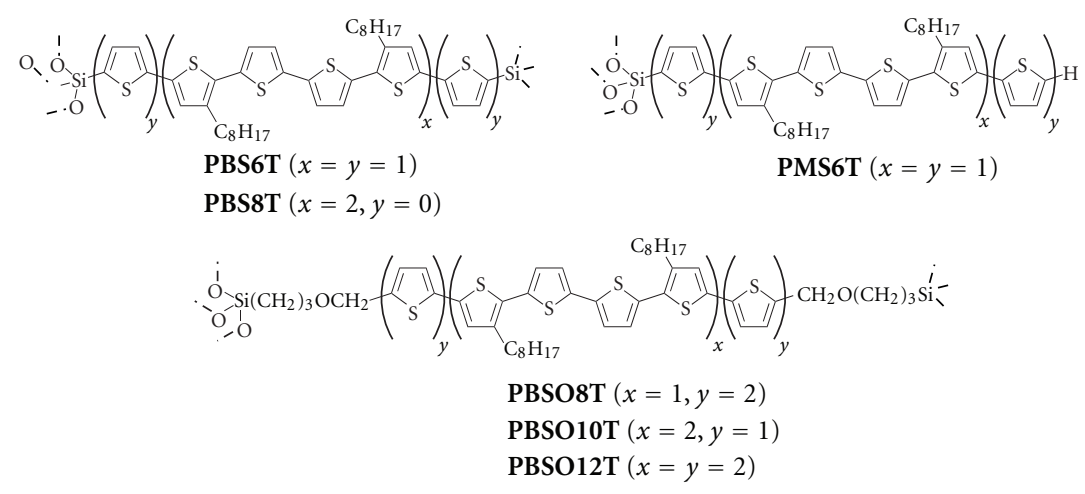

FIgURE 1: Chemical structures of PSQs having oligothiophenes.

[12-14] using a coupling reaction between triethoxychlorosilane and lithiated oligothiophenes obtained by the reaction of oligothiophenes and $n$-butyllithium (Scheme 2). The crude products mainly contained $\mathbf{B S} n \mathbf{T}(n=6$ and 8 ) and they could be purified by preparative GPC. Small amount of mono(triethoxysilyl)-substituted oligothiophenes (MSnT) was isolated as a major byproduct.

2.2. Indirect Silylation. We tried also to synthesize bis(triethoxysilyl)-substituted deca- and dodecathiophene in the same manner as shown in Scheme 2. However, the reactivity of these oligothiophenes with $n$-butyllithium was low, and the lithiation reaction did not proceed at $-78^{\circ} \mathrm{C}$. Although the reaction was made at room temperature to increase the reactivity, the selectivity of lithiation became low, and bulky triethoxysilyl groups were introduced not only at $\alpha$-positions but also at $\beta$-position of thienylene units. The formation of multisilylated oligothiophenes is unfavorable for developing electrically highly conductive materials because the steric hindrances may reduce effective $\pi$-conjugation lengths of oligothiophenes.
We have designed a new synthetic pathway, as shown in Scheme 3, in order to introduce triethoxysilyl groups only at $\alpha$-positions of both terminal thienyl groups of oligothiophenes [15]. Although Scheme 3 consists of much more reaction steps than Scheme 2, we have found that silyl groups could be surely introduced only at the $\alpha$-position of terminal thienyl groups, and reaction steps included in this route proceed with reasonable yields.

\section{Polymerization of Monomers and Anchoring of Polymers}

Polycondensation process of monomers monitored by GPC is shown in Figure 2. When a dilute hydrochloric acid $(0.1 \mathrm{M})$ as a polymerization catalyst was added into the THF solution of monomers, the molecular weight of the formed polymers started to increase soon after the addition of the acid catalyst and leveled off at $6 \mathrm{hr}$, although the reaction solution remained homogenous. However, once the solvent was removed from the polymer solution, orange or reddishorange solids which were insoluble in common organic 


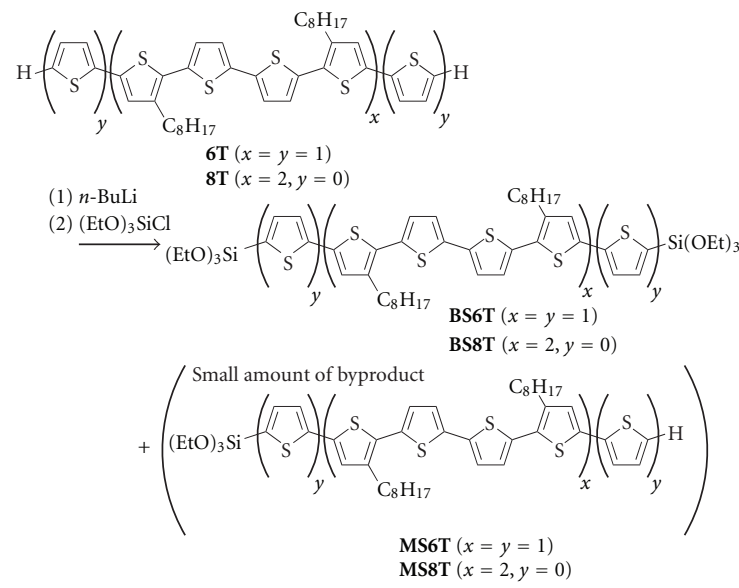

Scheme 2: Synthesis of BSnT and MSnT ( $n=6$ and 8).
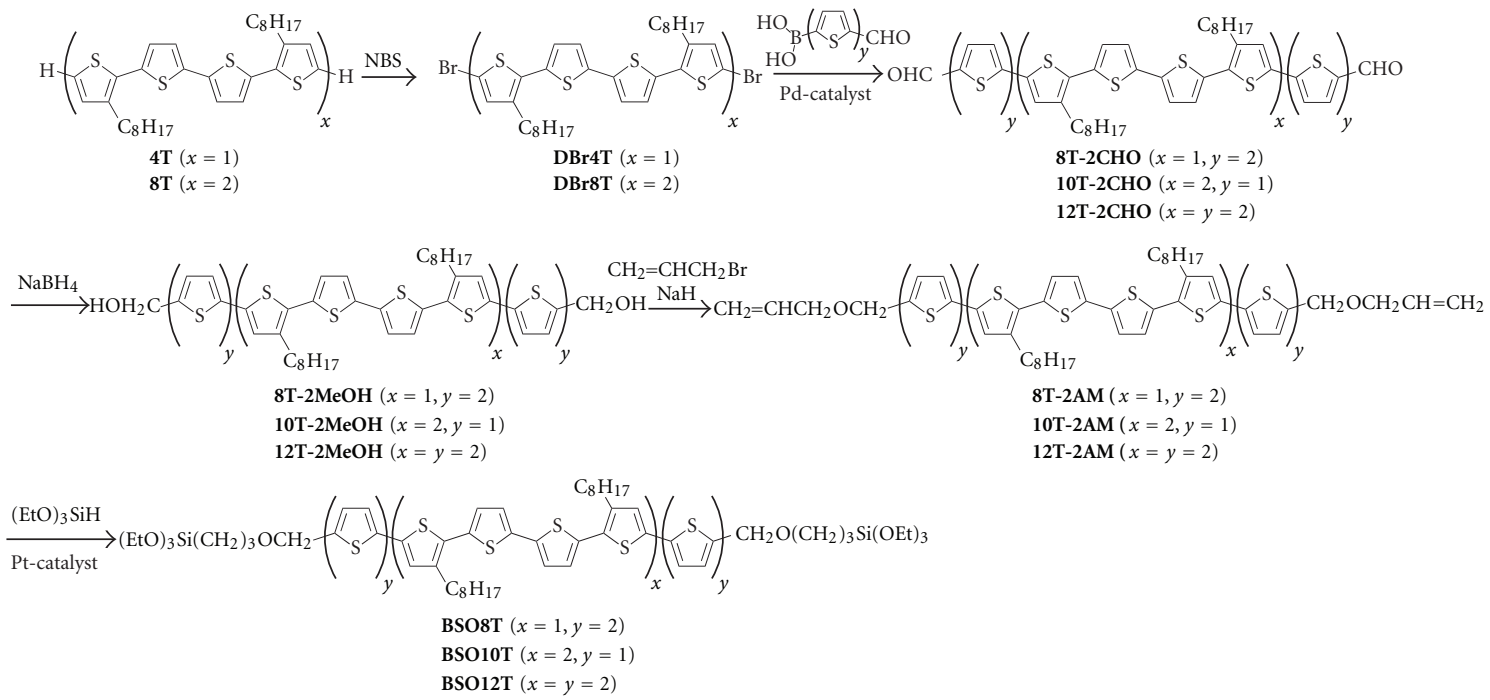

Scheme 3: Synthesis of BSOnT ( $n=8,10$, and 12).

solvents were obtained. The FT-IR spectra of polymers showed a broad and strong peak at $1000-1200 \mathrm{~cm}^{-1}$ due to an $\mathrm{Si}-\mathrm{O}-\mathrm{Si}$ stretching vibration, which was not observed in the FT-IR spectra of monomers. These findings suggest, first, that the polymerization proceeds by the sol-gel reaction of the triethoxysilyl group to form a siloxane-bond network and, second, that the polymers in THF still have active sites which can react partly with hydroxyl groups on the surface of glass or ITO substrate, leading to the formation of insoluble polymer films fixed covalently on the substrate. The insoluble nature of polymer films is of a great use for the fabrication of multilayered molecular devices by a wet process. PSQs obtained from the polymerization reaction are named hereafter as PBSnT ( $n=6$ and 8), PMS6T, and PBSO $n$ T $(n=8,10$ and 12) (Figure 1).

To confirm the role of the PSQ network, the polymer film in class I was prepared by the sol-gel method using a mixture of tetraethoxysilane (TEOS) and octithiophene $(\mathbf{8 T})$, and the morphology of the class I film was compared with that of PBS8T (class II film). In the class I film, the sea-and-island morphology was observed due to the phase separation between $8 \mathrm{~T}$ (island) and $\mathrm{SiO}_{2}$ from TEOS (sea) (Figure 3(b)). The phase separation is not favorable for electrical conduction because a conducting pathway will be segmented due to the isolation of conductive oligothiophene phases in the insulating $\mathrm{SiO}_{2}$ matrix. It was also found that $8 \mathrm{~T}$ was removed readily by dipping the solgel film into acetone, showing that the $\mathbf{8 T}$ molecules were just embedded in $\mathrm{SiO}_{2}$ matrix without forming covalent bonds. In contrast, the PBS8T film was very smooth and homogeneous (Figure 3(a)) and was insoluble in common organic solvents.

\section{Electrochemical Properties}

Stability of the polymer films was examined by cyclic voltammetry (CV) in tetraethylammonium perchlorate (TEAP) 


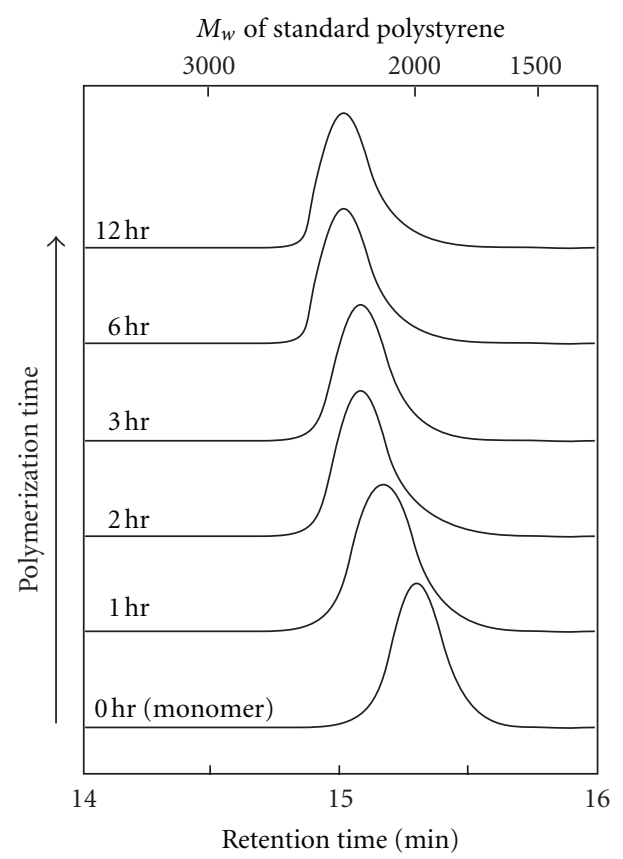

FIGURE 2: GPC curves of reaction solutions during polymerization of BS8T.

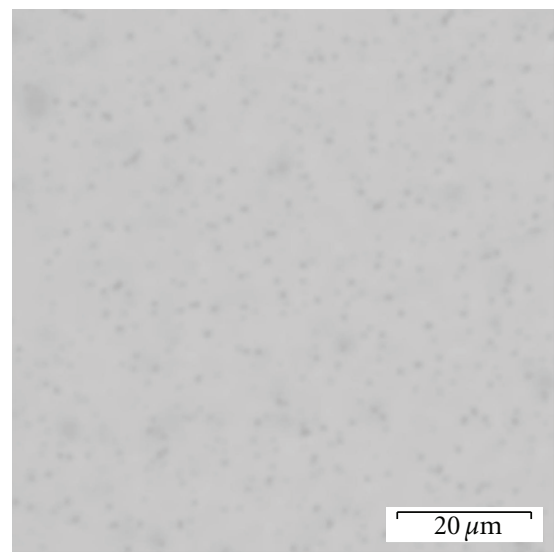

(a)

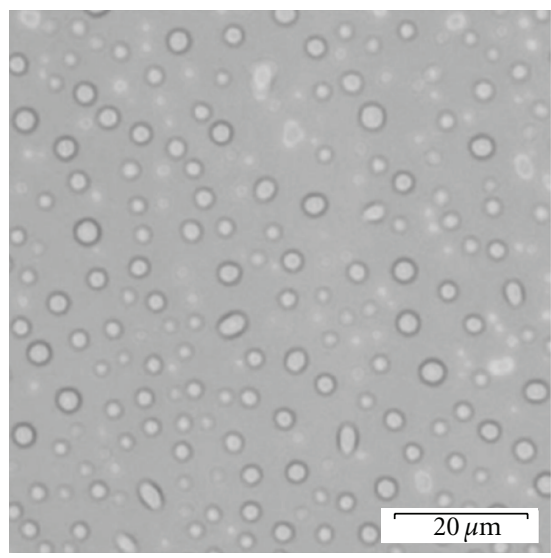

(b)

FIGURE 3: Optical microscope images of (a) PBS8T film and (b) film obtained from TEOS and 8T.

(0.1 M)/acetonitrile (Figure 4). The PBS8T film exhibited broad oxidation peaks at 0.6 and $0.8 \mathrm{~V}$ versus $\mathrm{Ag} / \mathrm{Ag}^{+}$, while two oxidation peaks were observed at 0.5 and $0.7 \mathrm{~V}$ for the PBSO8T film. Four alkyl groups are introduced in octithiophene moiety in PBS8T, compared with two alkyl groups for PBSO8T. By this difference, $\pi$-conjugation length of octithienylene units in PBSO8T will be well expanded because of less stertic hindrance, which may cause the negative shift of the oxidation potential relative to those of PBS8T. PBSO10T also showed two oxidation peaks centered at 0.3 and $0.6 \mathrm{~V}$, but PBSO12T showed a broad oxidation peak in the potential range from 0.2 to $0.7 \mathrm{~V}$.

Although PBS6T showed an irreversible CV curve and its current density gradually decreased by the repeated potential cycling, other PSQ films (PBS8T and PBSOnT) showed broad but reversible redox waves, suggesting the electrochemical stability of oligothiophenes longer than hexamer. Corriu et al. reported the synthesis and electrochemical properties of PSQs having oligothiophenes with short $\pi$-conjugation units (monomer to trimer) [16]. In their report, when the polymers were chemically or electrochemically oxidized, oligothiophene moieties were eliminated and polymerized due to the poor stability of the oxidized species. In contrast to their results, PBS8T and PBSOnT showed a good electrochemical stability due to delocalization of charges formed on the well expanded $\pi$-conjugation unit and a good adhesion of the polymer to the ITO substrate. 


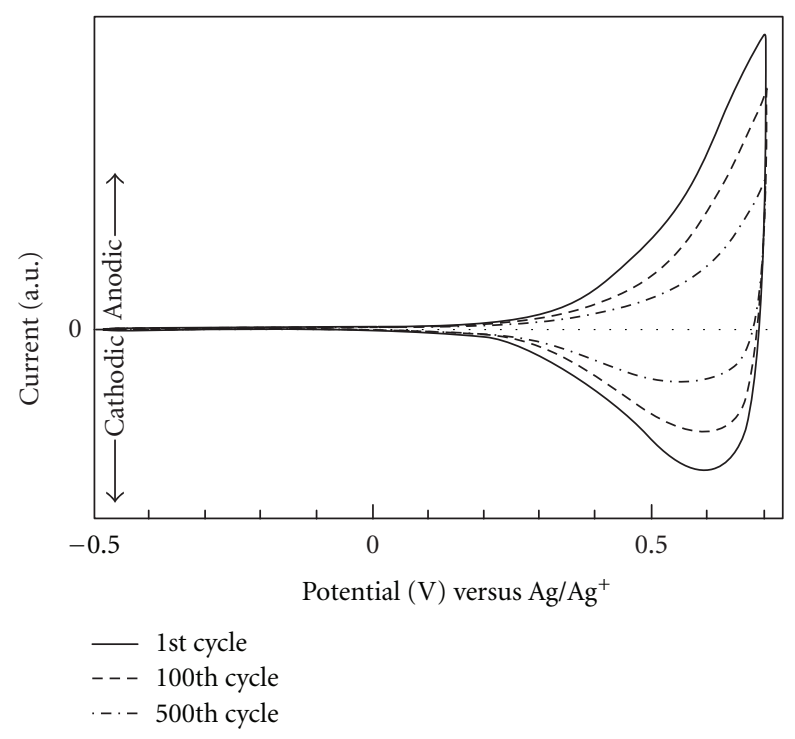

(a)

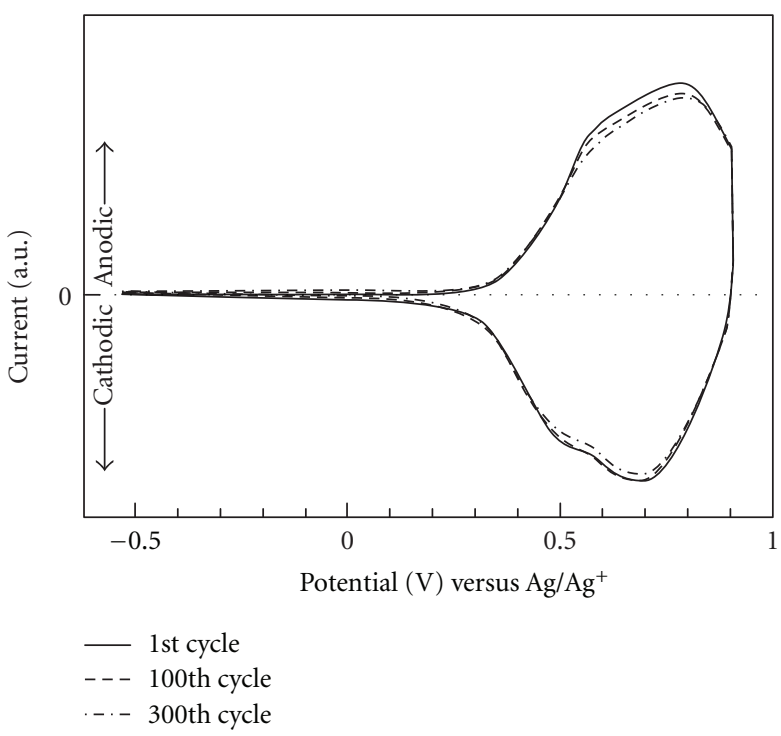

(b)

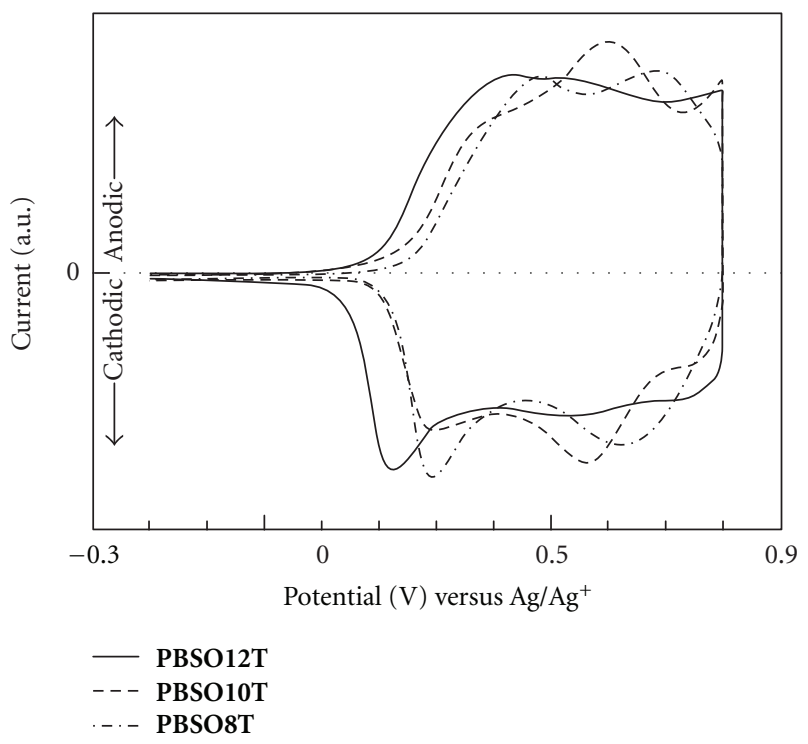

(c)

FIgure 4: Cyclic voltammograms of (a) PBS6T, (b) PBS8T, and (c) PBSOnT ( $n=8,10$, and 12) films in TEAP (0.1 M)/acetonitrile at a scan rate of $50 \mathrm{mV} \mathrm{s}^{-1}$.

\section{Spectroelectrochemistry}

Absorption spectra of PSQ films biased at different potentials were measured in TEAP $(0.1 \mathrm{M})$ /acetonitrile to identify chemical species to be formed at different oxidation states of PSQs (Figure 5, Table 1). As shown in the inset of Figure 5, the neutral PBS8T film showed a single absorption band at $445 \mathrm{~nm}$ due to $\pi-\pi^{*}$ transition of a neutral octithiophene moiety. When the PBS8T film was oxidized at $0.6 \mathrm{~V}$, the intensity of the 445-nm band decreased, and instead two absorption bands appeared at 749 and $1204 \mathrm{~nm}$, which were ascribed to the one-electron oxidized species (monocation radical or $\pi$-dimer) of octithienylene unit. The PBS8T film oxidized at $0.9 \mathrm{~V}$ was almost colorless and showed a single broad band at $970 \mathrm{~nm}$, being ascribed to the two-electron oxidized species (dication) of octithienylene unit. Similar results were obtained with PBSO8T. The $\pi-\pi^{*}$ absorption bands in the neutral PBSO10T and PBSO12T were red-shifted relative to those of PBS8T and PBSO8T, reflecting that the effective $\pi$-conjugation length of deca- and dodecathiophene is longer than that of octithiophene. Furthermore, the absorption bands of their oxidized species in the polymers were also red-shifted with increasing the number of thiophene rings. In all cases except PBS6T, polymer films could be one- and two-electron oxidized in the potential range from 0 to $0.9 \mathrm{~V}$ versus $\mathrm{Ag} / \mathrm{Ag}^{+}$. 


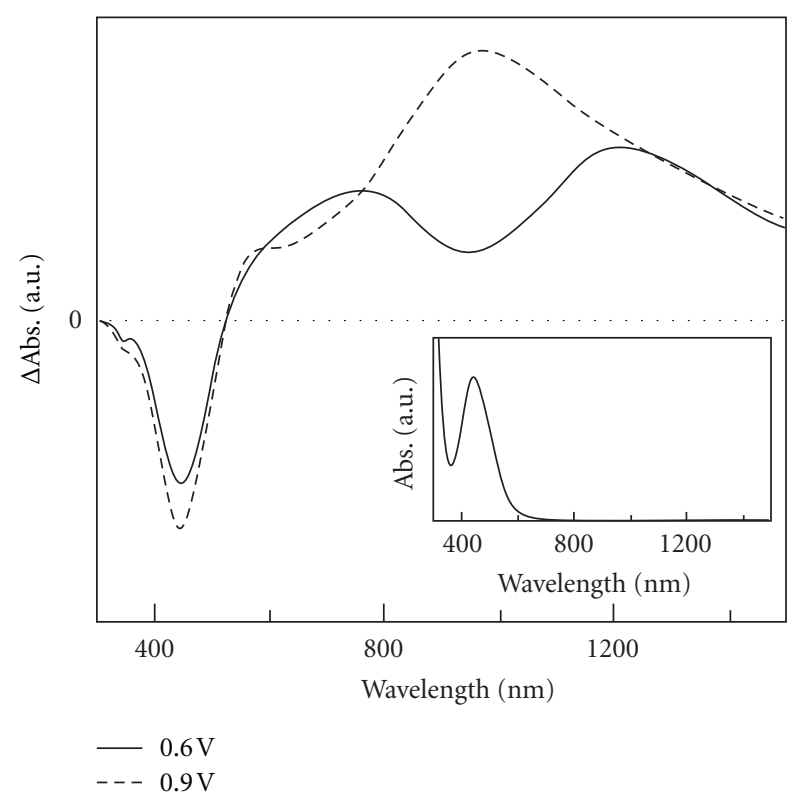

Figure 5: Difference absorption spectra of PBS8T film at 0.6 and $0.9 \mathrm{~V}$ referred to spectrum of neutral PBS8T film. The inset shows absorption spectrum of neutral PBS8T film.

TABLE 1: Absorption maxima of neutral and oxidized polymers.

\begin{tabular}{lccc}
\hline Polymers & Neutral state & $\begin{array}{c}\text { Wavelength/nm } \\
\text { One-electron oxidized state }\end{array}$ & Two-electron oxidized state $^{\mathrm{b}}$ \\
\hline PBS6T & 435 & $-^{\mathrm{c}}$ & c $^{\mathrm{c}}$ \\
PBS8T & 445 & 749,1204 & 970 \\
PBSO8T & 447 & 697,1211 & 1099 \\
PBSO10T & 482 & 708,1335 & 1252 \\
PBSO12T & 486 & 752,1405 & 1247 \\
\hline
\end{tabular}

${ }^{a}$ Oxidized at 0.6 V for PBS8T, at 0.5 V for PBSO8T, and at 0.4 V for PBSO10T and PBSO12T.

${ }^{\mathrm{b}}$ Oxidized at $0.9 \mathrm{~V}$ for PBS8T and at $0.8 \mathrm{~V}$ for PBSOnT.

${ }^{\mathrm{c}}$ Not observed.

\section{Electrical Properties}

Doping levels and electrical conductivities were measured by using an in situ electrochemical technique (Figure 6). In concert with the electrochemical oxidation process of the PBS8T film (Figure 4), the doping level gradually increased and finally reached around $25 \%$ at $0.9 \mathrm{~V}$, which corresponds to $200 \%$ doping per octithienylene unit and suggests that all octithienylene units are completely twoelectron oxidized at $0.9 \mathrm{~V}$. The plot of $\log$ (doping level) versus potential fits a straight line in a low doping region, and the inverse of its slope value is around $100 \mathrm{mV} /$ decade, which is larger than $60 \mathrm{mV} /$ decade for a common oneelectron transfer process at room temperature. We have already revealed that the slope value is a measure of distribution of effective $\pi$-conjugation lengths in conjugated oligomers and polymers [17-19]. In the PBS8T film, since both terminal thiophene rings of octithienylene unit are tightly fixed with silsesquioxane network, their conformation is partially restricted not to spread the $\pi$-conjugation all over the oligothienylene unit (vide infra). The electrical conductivity also increased with increasing the electrode potential from 0.3 to $0.7 \mathrm{~V}$, showed a maximum value of $5.6 \times$ $10^{-3} \mathrm{~S} \mathrm{~cm}^{-1}$, and then decreased to $3.0 \times 10^{-3} \mathrm{~S} \mathrm{~cm}^{-1}$ at $0.9 \mathrm{~V}$ (Figure 6(b)).

The doping levels and electrical conductivities of PBSO $n$ T ( $n=8,10$, and 12) changed with the electrode potential similarly to those of PBS8T. The electrical conductivities of PBSOnT increased with the increase in the chain length of oligothienylene units in PSQs.

To get an insight into charge transport properties of the polymer films, apparent mobilities of charge carriers were estimated by combining doping level and conductivity data. The mobilities are plotted in Figure 7 as a function of doping level. In all cases, the mobilities at the low doping level below $1 \%$ are $1-2 \times 10^{-6} \mathrm{~cm}^{2} \mathrm{~V}^{-1} \mathrm{~s}^{-1}$, which can be explained by the interchain hopping transport of monocation radicals (polarons). The mobility plots showed maxima at doping levels of $10 \%-15 \%$, in which oneand two-electron oxidized states coexist. When the doping level was increased beyond 20\%, the mobilities of PBS8T and PBSO8T greatly decreased, while the mobilities of 


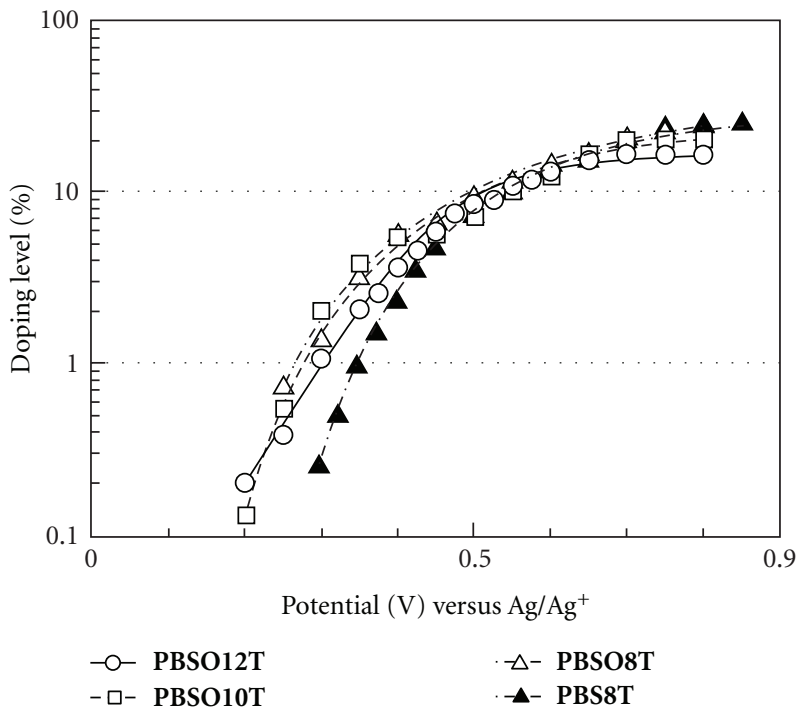

(a)

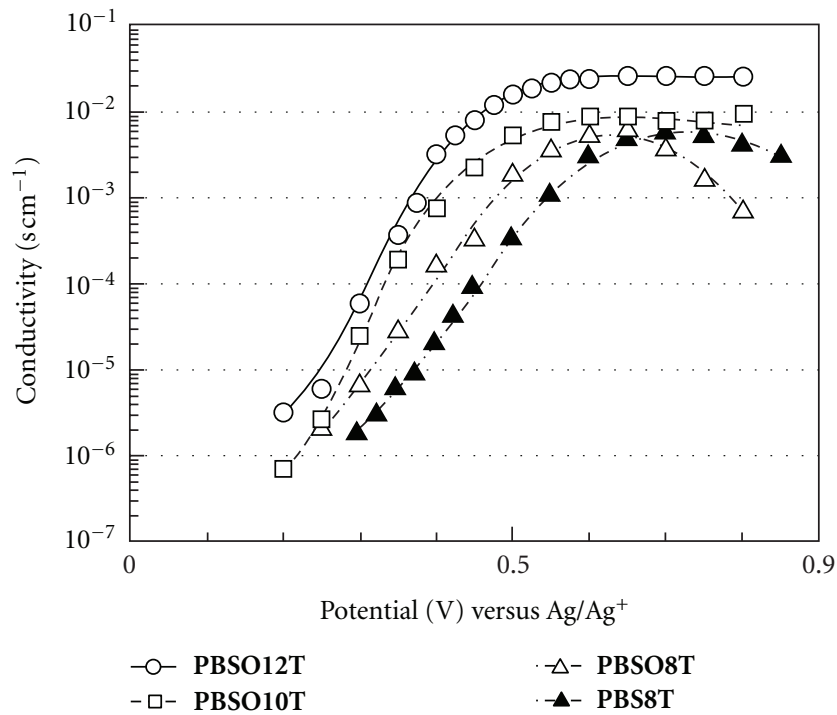

(b)

FIGURe 6: (a) Doping levels and (b) electrical conductivities plotted against electrode potential for $\boldsymbol{\Delta}$ : PBS8T, $\triangle$ : PBSO8T, $\square$ : PBSO10T, and $\bigcirc$ : PBSO12T films.

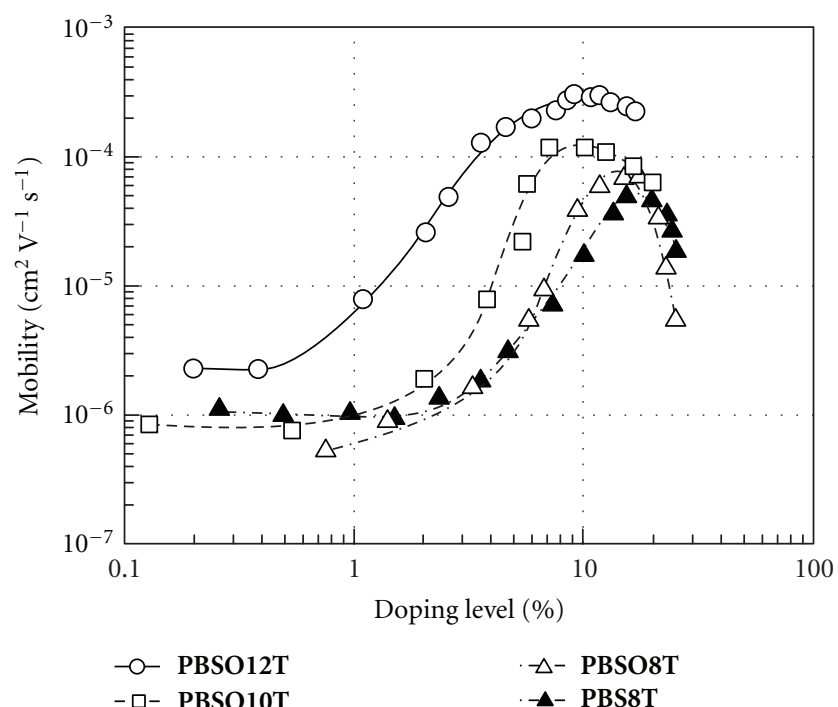

Figure 7: Apparent mobilities of charge carriers plotted against doping level for $\Delta$ : PBS8T, $\triangle$ : PBSO8T, $\square$ : PBSO10T, and $\bigcirc$ : PBSO12T films.

PBSO10T and PBSO12T did not decrease considerably. A plausible reason for this difference may be explained by the length of oligothienylene unit in the polymer; almost all oligothienylene units are two electrons oxidized at a doping level of $20 \%$, so that it becomes difficult for the positive charges to move among the oligothienylene units because of a Coulombic repulsion. However, the degree of the Coulombic repulsion will become smaller when the chain length of oligothienylene unit is longer. Thus, the degree of decrease of the mobilities becomes smaller with the increase of the number of thiophene rings.

\section{Effect of Silsesquioxane Network}

The mechanical hardness of the PSQ films was measured by a pencil hardness test according to the ASTM Standard D 3363-92 [20] and the results are summarized in Table 2. For the comparison, monomer films were prepared on glass or ITO substrate by spin-coating of a BSOnT solution of THF containing no polymerization catalyst [15]. The PBSO12T film did not show any damages by being scratched with $4 \mathrm{H}$ pencil, while the BSO12T film showed some damages even by B pencil. Similar results were obtained with other PSQ films. In the cases of PEDOT-PSS and common polymer films such as poly(styrene) (PSt) and poly(methyl methacrylate) (PMMA), appreciable damages were caused even by soft pencils (2B for PEDOT-PSS and PSt, 2H for PMMA). These results demonstrate clearly that the mechanical hardness of the PSQ films is much higher than those of trialkoxysilane monomers and other type of polymers having no chemical linkage with substrates, indicative of a hard network structure of silsesquioxane polymers and adhesion to metal oxides.

Figure 8 shows the absorption spectra of BSO12T and PBSO12T films in the neutral states. While the PBSO12T film exhibits a broad structureless spectrum with $\lambda_{\max }=$ $486 \mathrm{~nm}$, the spectrum of the BSO12T film displays a vibronic fine structure. Also, the absorption tail of BSO12T is redshifted by about $15 \mathrm{~nm}$ relative to that of PBSO12T. This redshift suggests the expansion of the effective $\pi$ conjugation length of the oligothienylene unit in BSO12T, where the oligothiophene units are not restricted by the siloxane network. 
TABLE 2: Results of pencil hardness test for PBSO12T, BSO12T, PMMA, PSt, and PEDOT-PSS.

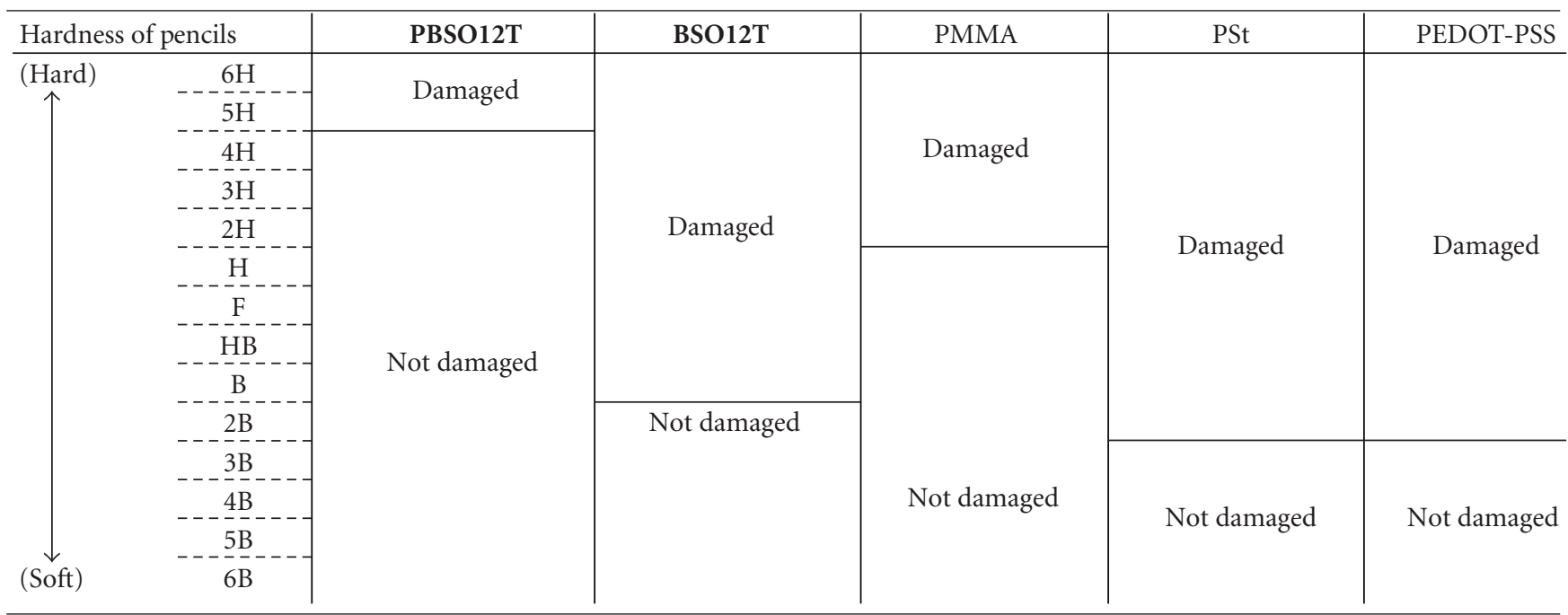

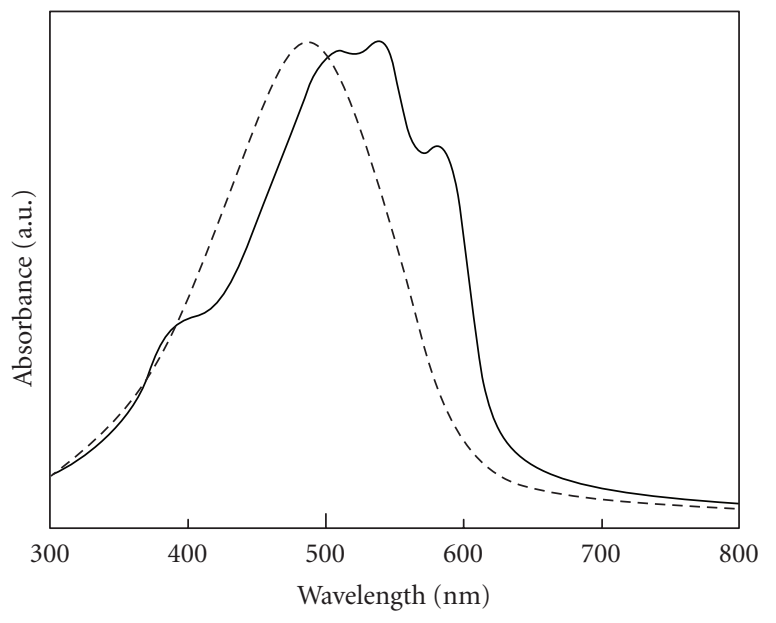

$\begin{array}{ll}- & \text { BSO12T } \\ --- & \text { PBSO12T }\end{array}$

Figure 8: Absorption spectra of neutral BSO12T and PBSO12T films.

A clear difference was seen also in cyclic voltammograms of BSO12T and PBSO12T films in TEAP $(0.1 \mathrm{M})$ /acetonitrile (Figure 9$)$. The cyclic voltammogram of the BSO12T film shows a sharp oxidation peak at $0.25 \mathrm{~V}$ versus $\mathrm{Ag} / \mathrm{Ag}^{+}$, compared with a broad oxidation peak for the PBSO12T film. The difference in shape of the cyclic voltammograms is reflected in the plots of $\log$ (doping level) versus potential shown in Figure 10. The inverse of the slope value of the plot in the low potential region is around $130 \mathrm{mV} /$ decade for the PBSO12T, much greater than $80 \mathrm{mV} /$ decade for the BSO12T. The value of $80 \mathrm{mV} /$ decade for the BSO12T monomer film is slightly greater than the theoretical value of $60 \mathrm{mV} /$ decade for a common oneelectron transfer process at room temperature, suggesting that the effective $\pi$-conjugation length of oligothienylene unit is somewhat distributed [18]. From the difference

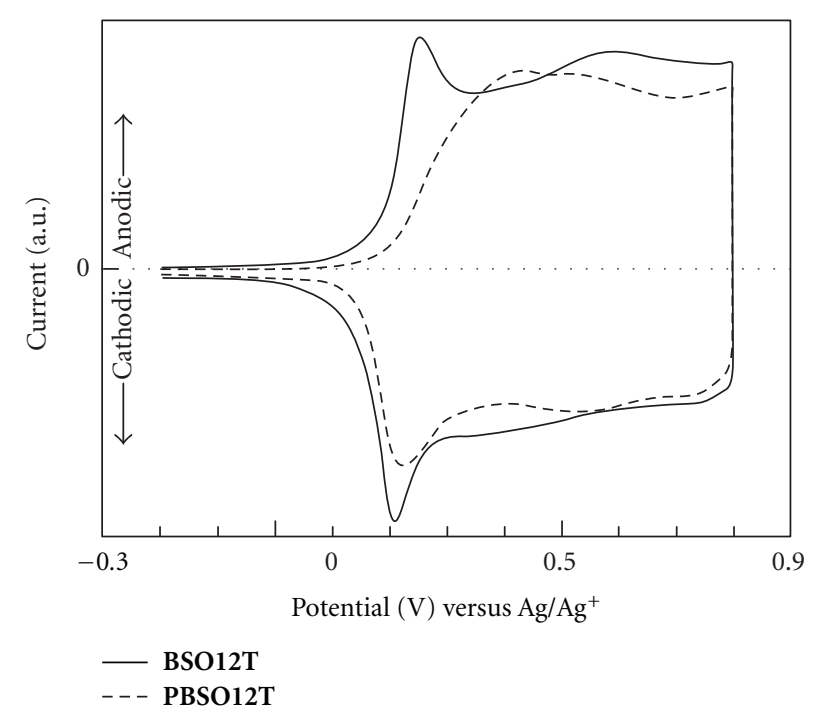

FIgure 9: Cyclic voltammograms of BSO12T and PBSO12T films in $\operatorname{TEAP}(0.1 \mathrm{M}) /$ acetonitrile at a scan rate of $50 \mathrm{mV} \mathrm{s}^{-1}$.

in the slope value, one can conclude that the effective $\pi$-conjugation length in the PBSO12T polymer film is distributed much wider than that in the BSO12T monomer film. It is likely that this may be caused by the rigid nature of the siloxane network.

Since we obtained a mono(triethoxysilyl)-substituted monomer, MS6T, as a byproduct by chance during the synthesis of BS6T, we attempted the polymerization of MS6T to obtain PMS6T (the chemical structure is shown in Figure 1), where one terminal of sexithiophene moiety is linked to silsesquioxane polymer. Compared with PBS6T, the cyclic voltammogram of PMS6T showed a clear redox wave (Figure 11), suggesting the higher flexibility of sexithienylene unit in the polymer film. This finding also supports the rigid siloxane network of PSQs. 


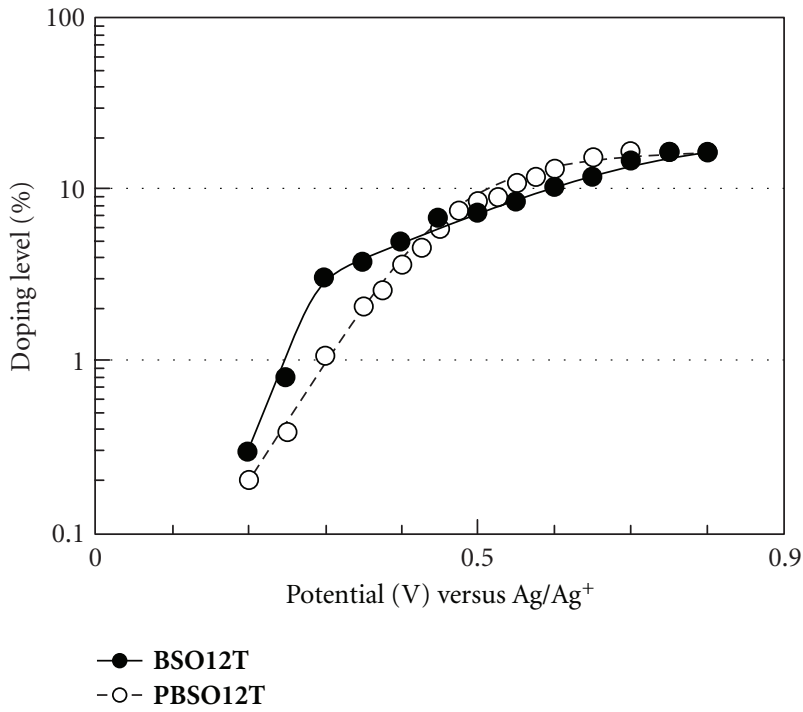

Figure 10: Doping levels plotted against electrode potential for BSO12T and $\bigcirc$ : PBSO12T films.

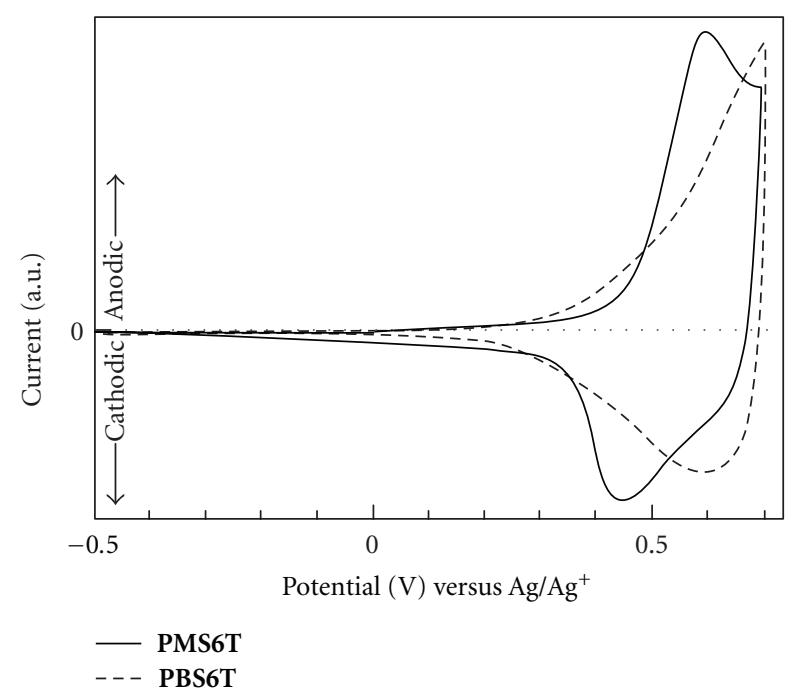

Figure 11: Cyclic voltammograms of PMS6T and PBS6T films in $\operatorname{TEAP}(0.1 \mathrm{M}) /$ acetonitrile at a scan rate of $50 \mathrm{mV} \mathrm{s}^{-1}$.

It was found that the maximum conductivity of BSO12T was $4.0 \times 10^{-1} \mathrm{~S} \mathrm{~cm}^{-1}$, which was by one order of magnitude greater than that of PBSO12T (Figure 12). There may be two plausible reasons for the difference in conductivity between BSO12T and PBSO12T. The first reason originates from the positional disorder. Silsesquioxane polymer may be amorphous, so that the position of oligothiophenes in the polymer is disordered. Thus, the pathway of the charge transport will become much longer than the case for the monomer film. The second reason arises from the energetic disorder. As mentioned above, the conformation is partially restricted not to spread the $\pi$-conjugation all over the oligothienylene unit. Thus, oligothienylene units with different effective $\pi$-conjugation length coexist in the

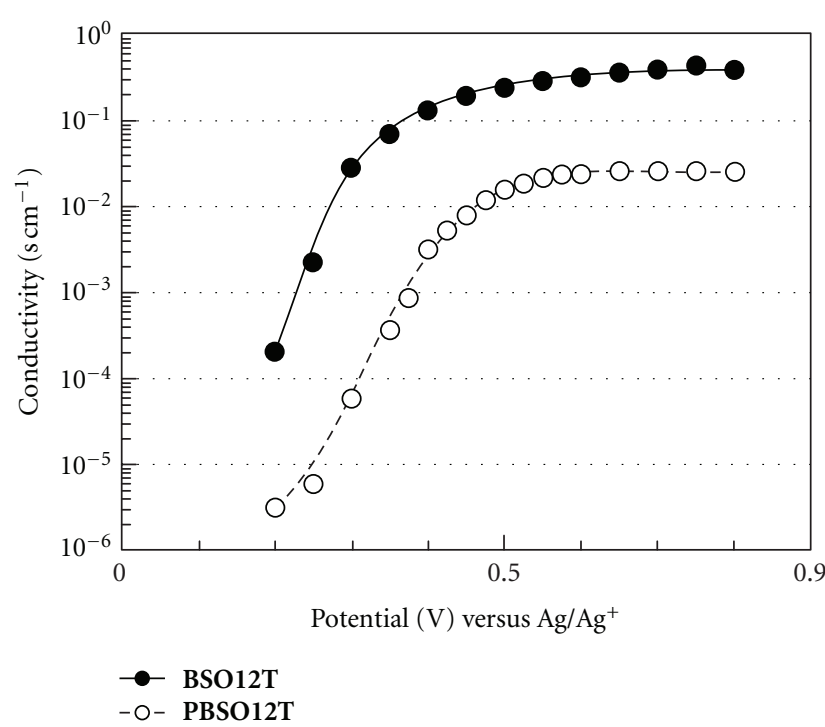

FIGURE 12: In situ electrical conductivities plotted against electrode potential for : BSO12T and $\bigcirc$ : PBSO12T films.

polymer, which means that some of oligothienylene site will act as trapping sites for the charge transport.

A study on the modification on polymerization conditions and polymer structures is currently under way to enhance the effective $\pi$-conjugation length, leading to higher conductivities, by controlling the flexibility of the silsesquioxane network. For example, we have found that 3,4-ethylenedioxythiophene (EDOT) unit can enhance the $\pi$-conjugation length, and an EDOT-containing polymer showed an excellent electrical conductivity [21]. So, EDOTcontaining oligothiophenes will become good candidates as oligothienylene units in PSQs.

\section{Summary}

Synthesis and physicochemical properties of polysilsesquioxanes incorporating oligothiophenes were reviewed. We found that with increasing the chain length of oligothienylene units in the polymers, Coulombic repulsion between oxidized polymer chains became smaller, the absorption bands of neutral and oxidized films showed red-shift, and the electrical conductivity and charge carrier mobility increased. The comparison with the monomer film revealed that the siloxane network enhanced the mechanical hardness of the polymer film, but its rigidity restricted a conformational change of oligothienylene units, leading to low conductivities of the PSQ films.

\section{Acknowledgments}

This work was supported in part by a Grant-in-Aid for scientific research from the Japan Society for the Promotion of Science (JSPS) (no. 22550198) and Adaptable and Seamless Technology Transfer Program through Target-Driven R\&D (A-STEP) from the Japan Science and Technology Agency 
(JST), the Furukawa Technology Promotion Foundation, and the Hitachi Metals-Materials Science Foundation.

\section{References}

[1] N. Auner and J. Weis, Organosilicon Chemistry VI, from Molecules to Materials, Wiley-VCH, Weinheim, Germany, 2005.

[2] S. J. Clarson, J. J. Fitzgerald, M. J. Owen, S. D. Smith, and M. E. Van Dyke, Science and Technology of Silicones and SiliconeModified Materials, ACS Symposium Series 964, Oxford University Press, Washington, DC, USA, 2007.

[3] C. Sanchez and F. Ribot, "Design of hybrid organic-inorganic materials synthesized via sol-gel chemistry," New Journal of Chemistry, vol. 18, no. 10, pp. 1007-1047, 1994.

[4] K. J. Shea and D. A. Loy, "Bridged polysilsesquioxanes. Molecular-engineered hybrid organic-inorganic materials," Chemistry of Materials, vol. 13, no. 10, pp. 3306-3319, 2001.

[5] A. Walcarius, "Electrochemical applications of silica-based organic-inorganic hybrid materials," Chemistry of Materials, vol. 13, no. 10, pp. 3351-3372, 2001.

[6] R. Corriu, "Organosilicon chemistry and nanoscience," Journal of Organometallic Chemistry, vol. 686, no. 1-2, pp. 32-41, 2003.

[7] A. Walcarius, D. Mandler, J. A. Cox, M. Collinson, and O. Lev, "Exciting new directions in the intersection of functionalized sol-gel materials with electrochemistry," Journal of Materials Chemistry, vol. 15, no. 35-36, pp. 3663-3689, 2005.

[8] F. Lerouge, G. Cerveau, and R. J. P. Corriu, "Supramolecular self-organization in non-crystalline hybrid organic-inorganic nanomaterials induced by van der Waals interactions," New Journal of Chemistry, vol. 30, no. 10, pp. 1364-1376, 2006.

[9] D. Fichou, Ed., Handbook of Oligo- and Polythiophenes, WileyVCH, Weinheim, Germany, 1999.

[10] P. Bäuerle, "Oligothiophenes," in Electronic Materials: the Oligomer Approach, K. Müllen and G. Wegner, Eds., chapter 2, pp. 105-1197, Wiley-VCH, Weinheim, Germany, 1998.

[11] A. Mishra, C. Q. Ma, and P. Bäuerle, "Functional oligothiophenes: molecular design for multidimensional nanoarchitectures and their applications," Chemical Reviews, vol. 109, no. 3, pp. 1141-1276, 2009.

[12] I. Imae, S. Takayama, D. Tokita et al., "Development of anchored oligothiophenes on substrates for the application to the tunable transparent conductive films," Polymer, vol. 50, no. 26, pp. 6198-6201, 2009.

[13] Y. Harima, J. Ohshita, I. Imae, T. Sugioka, and K. Kanehira, "Electrically conductive laminates having organic compounds on surfaces and their manufacture, doped electrically conductive materials, and electrochromic materials," JP 2010266727 A 20101125, Japan Kokai Tokkyo Koho, 2010.

[14] I. Imae, D. Tokita, Y. Ooyama, K. Komaguchi, J. Ohshita, and Y. Harima, "Charge transport properties of polymer films comprising oligothiophene in silsesquioxane network," Polymer Chemistry, vol. 2, no. 4, pp. 868-872, 2011.

[15] I. Imae, D. Tokita, Y. Ooyama, K. Komaguchi, J. Ohshita, and Y. Harima, "Oligothiophenes incorporated in a polysilsesquioxane network: application to tunable transparent conductive films," Journal of Materials Chemistry, vol. 22, pp. 1640716415, 2012.

[16] R. J. P. Corriu, J. J. E. Moreau, P. Thepot, and M. W. C. Man, "Trialkoxysilyl Mono-, Bi-, and terthiophenes as molecular precursors of hybrid organic-inorganic materials," Chemistry of Materials, vol. 6, no. 5, pp. 640-649, 1994.
[17] Y. Harima, Y. Kunugi, K. Yamashita, and M. Shiotani, "Determination of mobilities of charge carriers in electrochemically anion-doped polythiophene film," Chemical Physics Letters, vol. 317, no. 3-5, pp. 310-314, 2000.

[18] J. Yano, M. Kobayashi, S. Yamasaki, Y. Harima, and K. Yamashita, "Mean redox potentials of polyaniline determined by chronocoulometry," Synthetic Metals, vol. 119, no. 1-3, pp. 315-316, 2001.

[19] Y. Harima, T. Eguchi, and K. Yamashita, "Enhancement of carrier mobilities in poly(3-methylthiophene) by an electrochemical doping," Synthetic Metals, vol. 95, no. 1, pp. 69-74, 1998.

[20] The American Society for Testing and Materials (ASTM) D, 3363- 92, Test Method for Film Hardness by Pencil Test.

[21] I. Imae, S. Imabayashi, K. Korai et al., "Electrosynthesis and charge-transport properties of poly $\left(3^{\prime}, 4^{\prime}\right.$-ethylenedioxy$2,2^{\prime}: 5^{\prime}, 2^{\prime \prime}$-terthiophene)," Materials Chemistry and Physics, vol. 131, no. 5, pp. 752-756, 2012. 

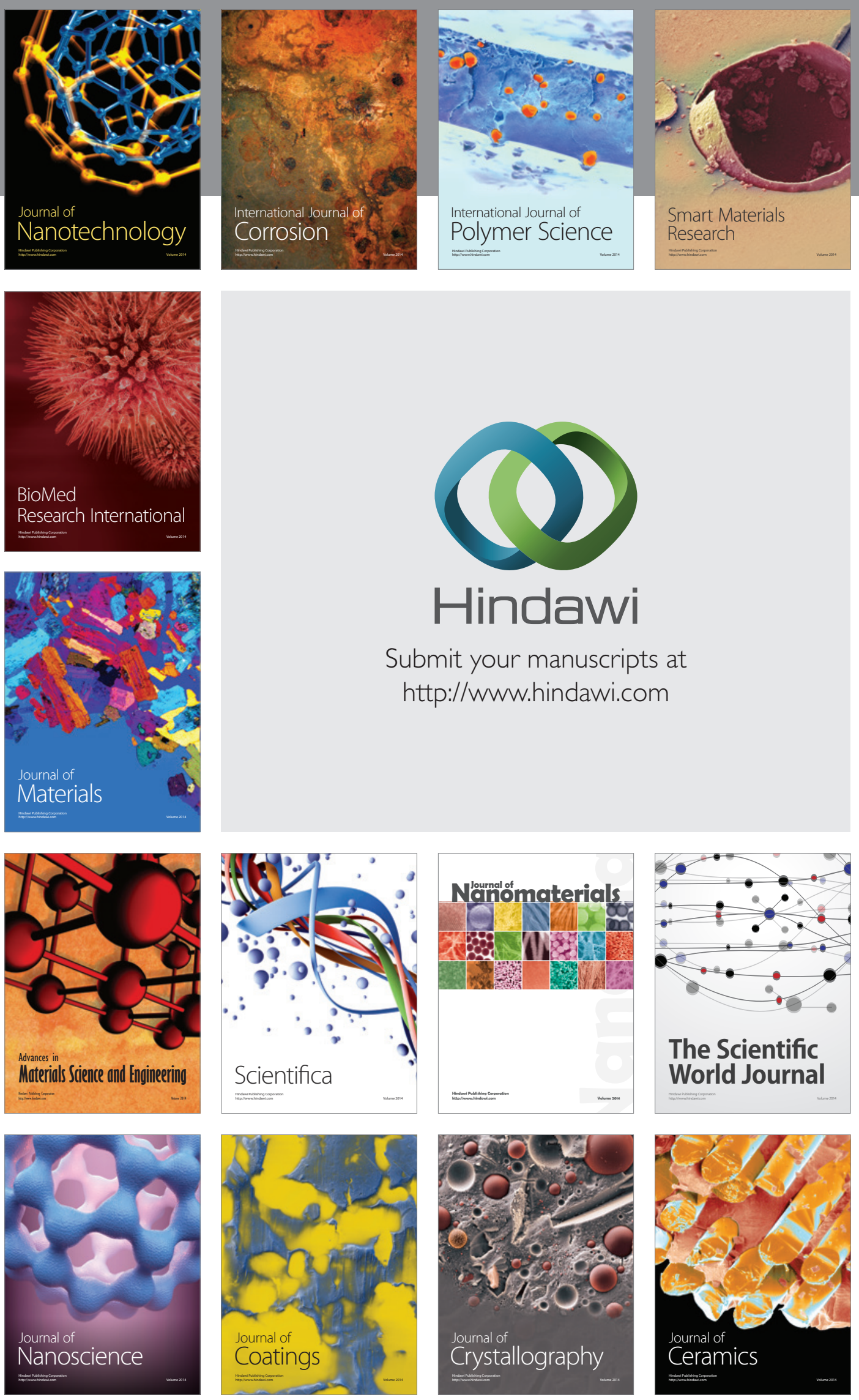

The Scientific World Journal

Submit your manuscripts at

http://www.hindawi.com

\section{World Journal}

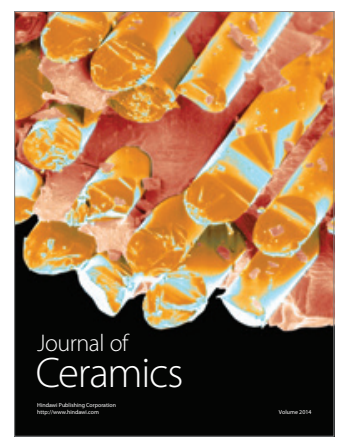

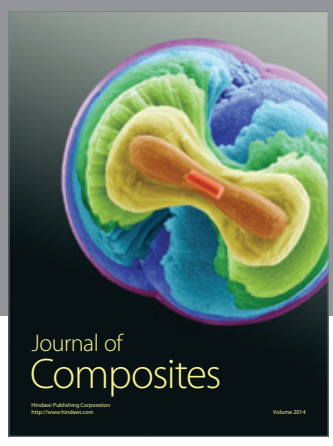
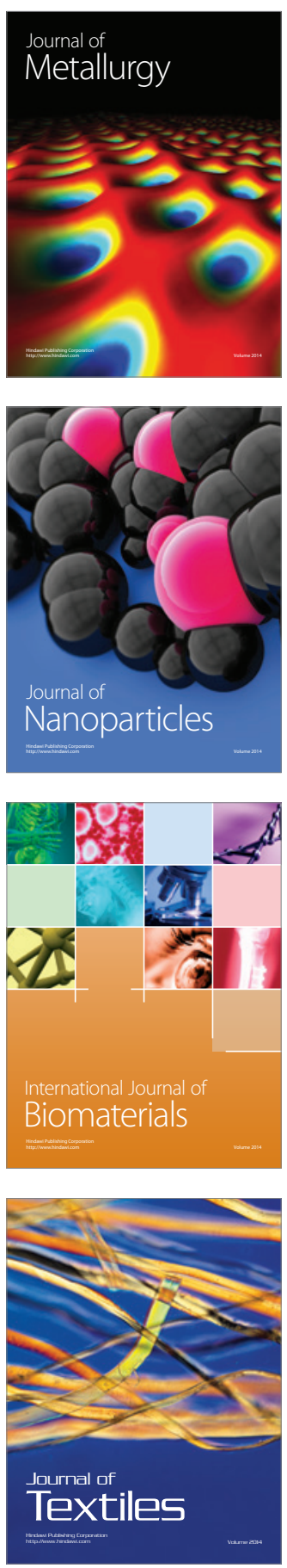